\title{
INCIDENCIA DE FUNGOS TOXIGÊNICOS E AFLATOXINAS EM ARROZ
}

\author{
Incidence of toxigenic fungi and aflatoxins in rice
}

\author{
Rosilane Aparecida de Carvalho ${ }^{1}$, Luís Roberto Batista ${ }^{2}$, Guilherme Prado ${ }^{3}$, \\ Bibiane Resende de Oliveira ${ }^{2}$, Daiani Maria da Silva ${ }^{2}$
}

\begin{abstract}
RESUMO
Um dos problemas mais sérios que confrontam a qualidade do arroz é a presença de fungos produtores de micotoxinas, principalmente as espécies pertencentes aos gêneros Aspergillus, Penicillium e Fusarium. Neste estudo, objetivou-se verificar os níveis de aflatoxinas e identificar a população fúngica associada a grãos de arroz beneficiado e comercializado em Belo Horizonte e algumas cidades do sul do estado. Foram analisadas um total de 60 amostras de arroz: orgânico, parboilizado, integral, polido, sendo a incidência de aflatoxinas verificada em uma amostra. Os resultados demonstraram que as espécies aflatoxigênicas identificadas foram A. parasiticus e A. flavus. Utilizando Cromatografia Líquida de Alta Eficiência para avaliar a incidência de aflatoxina em arroz, apenas uma apresentou contaminação de 1,2 ìg $\mathrm{Kg}^{-1}$. Apesar da presença de fungos aflatoxigênicos, as amostras não apresentaram níveis preocupantes de aflatoxinas que pudessem colocar em risco a segurança do produto e a saúde dos consumidores.
\end{abstract}

Termos para indexação: Aflatoxinas, alimentos, Aspergilus flavus, Aspergillus parasiticus.

\begin{abstract}
One of the most serious problems facing the quality of rice is the presence of mycotoxin-producing fungi, especially the species belonging to the genera Aspergillus, Penicillium and Fusarium. The objective of this study was to check the levels of aflatoxins and identify the fungal population associated with grains of rice received and sold in Belo Horizonte and some cities in the southern state. We analyzed a total of 60 samples of rice: organic, parboiled, full, polished, and the incidence of aflatoxins found in a sample. The results showed that the aflatoxigenic species identified were A. parasiticus and G2 and A. flavus. Using high performance liquid chromatography to assess the incidence of aflatoxin in rice, only one showed a contamination of $1.2 \mathrm{ig} \mathrm{kg}-1$. Despite the presence of aflatoxigenic fungi, the samples showed no worrying levels of aflatoxin that could endanger the safety of the product and consumer health.
\end{abstract}

Index terms: Aflatoxins, food, Aspergillus flavus, Aspergillus parasiticus.

(Recebido em 21 de novembro de 2008 e aprovado em 11 de fevereiro de 2010)

\section{INTRODUÇÃO}

$\mathrm{O}$ arroz (Oryzae sativa L) é um dos alimentos mais importantes produzidos em todo o mundo e sua qualidade e segurança estão diretamente relacionadas à presença de fungos produtores de micotoxinas. A incidência das espécies fúngicas varia de acordo com fatores bioclimáticos e com o tipo de produção Arnolds (1997). O conhecimento dessas espécies possibilita prever os riscos de contaminação e potencializar as pesquisas às eventuais micotoxinas presentes (Frisvad \& Samson, 1991). Dentre as micotoxinas, as aflatoxinas ocupam primariamente a classe das mais incidentes e mais tóxicas que se conhecem (Alddred et al., 2004). São produzidas principalmente pelas espécies do gênero Aspergillus pertencentes à Seção Flavi: Aspergillus flavus, A. parasiticus e A. nomius As espécies menos comum produtoras de aflatoxinas são $A$. pseudotamarii, A. bombycis e A. parvisclerotigenus (Klich, 2007). Quatro espécies não pertencentes à seção Flavi podem produzir aflatoxinas: A. ochraceoroseus, $A$. rambellii, Emericella venezuelensis e E. astellata (Frisvad et al., 2005). Neste estudo, objetivou-se identificar as espécies fúngicas e avaliar a incidência de aflatoxinas em amostras de arroz.

\section{MATERIAIS E MÉTODOS}

\section{Amostras}

As amostras de arroz de 1 a $5 \mathrm{Kg}$ foram adquiridas em estabelecimentos comerciais das seguintes cidades: Lavras, Ribeirão Vermelho, Perdões, Belo Horizonte e Ijaci, no período de março a dezembro de 2007 . Todas as amostras apresentavam bom estado de conservação e estavam dentro do prazo de validade, conforme indicado pelo fabricante Foram analisadas 60 amostras de arroz do grupo beneficiado subdivididas em quatro subgrupos: polido (48), parboilizado (3), integral (7) e orgânico (2), pertencentes às respectivas classes longo fino, longo e curto e tipos um, dois e três.

\footnotetext{
${ }^{1}$ Universidade Federal de Lavras/UFLA - Departamento de Ciência dos Alimentos/DCA - Cx. P. 3037 - $37200-000$ - Lavras, MG rosilane aparecida1976@yahoo.com.br

2Universidade Federal de Lavras/UFLA - Departamento de Ciência dos Alimentos/DCA - Lavras, MG

${ }^{3}$ Fundação Ezequiel Dias/FUNED - Laboratório de Micologia e Micotoxina - Belo Horizonte, MG
} 


\section{Isolamento e Identificação de fungos filamentosos}

Para o isolamento dos fungos presentes nas amostras de arroz, foi utilizada a técnica de Plaqueamento Direto em meio de cultura Dicloran Rosa de Bengala Clorafenicol (DRBC) com o plaqueamento de 100 grãos por amostra após desinfecção superficial, e incubação a $25^{\circ} \mathrm{C}$ por sete dias, conforme Samson et al. (2000). Os resultados foram expressos em porcentagem de partículas contaminadas. Os isolados das seções: Circundati, Flavi e Nigri foram identificados de acordo com o método de Klich (2002). Os gêneros Cladosporium e Fusarium de acordo com Pitt \& Hocking (1997) e Penicillium de acordo com Pitt (2000).

\section{Avaliação do Potencial Toxigênico dos fungos identificados:}

A técnica utilizada foi a Plug Agar, todos os isolados foram inoculados em meio de cultura YES (Yeast Extract Sucrose) e incubados por sete dias a $25^{\circ} \mathrm{C}$, conforme Filtenborg \& Frisvad (1980). Foram utilizadas placas de Cromatografia de Camada Delgada - Merck - Sílica Gel $20 \times 20 \mathrm{~cm}$ e a eluição ocorreu em uma cuba de vidro contendo TEF (Tolueno Acetato de Etila e Ácido Fórmico $90 \%$ - 50:40:10). A visualização foi feita a $366 \mathrm{~nm}$ por meio de Cromatovisor CAMAG (UV- Betrachter). Foram utilizados padrões de aflatoxinas B1, B2, G1, G2 (SigmaAldrich).

\section{Análise de aflatoxinas em arroz por CLAE}

As amostras de arroz foram analisadas por Cromatografia Líquida de Alta Eficiência (CLAE) descritas por Soares \& Rodriguez-Amaya (1988) e a quantificação por Stroka et al. (2000) a seguir: Extração: Cinquenta gramas da amostra foram pesadas em um Erlenmeyer de $500 \mathrm{~mL}$. Adicionou-se $270 \mathrm{~mL}$ de metanol p.a. e $30 \mathrm{~mL}$ de uma solução de cloreto de potássio $4 \%$. O frasco foi fechado com parafilme e papel alumínio e foi colocado em agitador mecânico tipo shaker sob agitação em velocidade média por 30 minutos. Após esse tempo o conteúdo do frasco foi filtrado em papel de filtro qualitativo e $150 \mathrm{~mL}$ do filtrado foram recolhidos em uma proveta graduada. Purificação: $\mathrm{O}$ filtrado foi transferido para um béquer de $600 \mathrm{~mL}$, e a ele adicionaram-se $150 \mathrm{~mL}$ de sulfato de cobre $10 \%$ e, aproximadamente, $50 \mathrm{~mL}$ de celite. Após homogeneização, a mistura foi filtrada em papel de filtro Whatman $n^{\circ} 1$ e 150 $\mathrm{mL}$ do filtrado foram recolhidos em uma proveta graduada. Partição Líquido-líquido: O filtrado recolhido foi transferido para um funil de separação ao qual foram adicionados 150 $\mathrm{mL}$ de água destilada e $10 \mathrm{~mL}$ de clorofórmio p.a.. O conteúdo do funil foi agitado vigorosamente por 3 minutos e, após a separação das fases, a fase clorofórmica foi recolhida em um frasco âmbar ambientada em clorofórmio. Esse procedimento foi repetido com mais 10 $\mathrm{mL}$ de clorofórmio. $\mathrm{O}$ extrato clorofórmico foi evaporado em banho maria a $50^{\circ}$.C sob atmosfera de nitrogênio. Os frascos foram então selados com parafilme, etiquetados e armazenados em a $-22^{\circ} \mathrm{C}$ até o momento da quantificação por cromatografia líquida de alta eficiência (CLAE). Quantificação por CLAE: O conteúdo dos frascos foi, então, retomado com $3 \mathrm{~mL}$ de solução metanol:água (2:3), submetido à agitação em agitador de tubos tipo vortex por aproximadamente 60 segundos e transferido para vial do auto amostrador do CLAE, após filtração em membrana de $13 \mathrm{~mm}$ de diâmetro e $0,45 \mu \mathrm{m}$ de porosidade. As amostras foram analisadas por CLAE (Cromatografia Líquida de Alta Eficiência) com detecção por fluorescência após derivação pós-coluna com brometo de potássio e Kobra Cell, usando um cromatógrafo líquido Shimadzu com detector de fluorescência (Shimadzu Modelo LC-10AD), excitação 420 nm, emissão 360 nm, com coluna Shim - Pack CLC - ODS, $5 \mu \mathrm{m}, 4,6$ × $250 \mathrm{~mm}$, precedido por guarda coluna Shim Pack G - ODS, $5 \mu \mathrm{m}, 4$ x $10 \mathrm{~mm}$. A fase móvel (filtrada em membrana $0,45 \mu \mathrm{m}$ e desgaseificada com hélio) usada foi acetonitrila: metanol: água $(2: 2: 6)$ acrescentada de ácido nítrico $4 \mathrm{~mol} / \mathrm{L}\left(350 \mu \mathrm{L} \mathrm{L}^{-1}\right)$ e brometo de potássio $(120 \mathrm{mg}$ $\left.\mathrm{L}^{-1}\right)$ a um fluxo de $1 \mathrm{~mL}$ por minuto. Uma curva padrão de aflatoxinas B1, B2, G1 e G2 foi preparada em concentrações que variaram de 79,2 a 0,47 ng/mL para $A F B 1,98,68$ a 0,59 $\mathrm{ng} / \mathrm{mL}$ para AFB2, 125,57 a $0,75 \mathrm{ng} / \mathrm{mL}$ para AFG1 e 79,10 a 0,48 para AFG2. Foram utilizados sete pontos na curva padrão, cada ponto, a partir da concentração inicial, possuía metade da concentração anterior. Injetou-se então $50 \mu \mathrm{L}$ das soluções padrão de aflatoxinas e dos extratos das amostras. A partir do cálculo da área dos picos das aflatoxinas dos extratos das amostras e das soluções padrões foi calculado o teor de aflatoxinas nas amostras. Nessas condições, o tempo de retenção foi de aproximadamente, 12,2 min., 15,1 min., 16,9 min. e 21,5 min. para as afaltoxinas G2, G1, B2 e B1, respectivamente.

\section{Eficiência da metodologia analítica para as aflatoxinas}

Para avaliar as características de desempenho da metodologia analítica para as aflatoxinas por CLAE, amostra de arroz, isenta de aflatoxinas, foi contaminada, em duplicata, em 5 concentrações de aflatoxinas B1, B2, G1 e G2.

\section{RESULTADOS E DISCUSSÃO}

\section{Contaminação por fungos filamentosos}

O resultado das análises demonstrou que, do total de amostras de arroz polido (47), 43 (92\%) apresentaram 
níveis de contaminação entre 1 e 42\%. Das amostras de arroz integral (7), cinco tiveram níveis de contaminação entre 4 e $32 \%$ e duas não tiveram contaminações detectadas. Das amostras de arroz orgânico (2), as duas apresentaram contaminações sendo 5 e 100\%. Em arroz parboilizado, as contaminações foram menores que $15 \%$. O número reduzido pode ser decorrente de boas práticas agrícolas e industriais. Os maiores índices de contaminação fúngica encontrados nas amostras de arroz analisadas estão listados a seguir: arroz orgânico (100\%), polido (42\%), integral (32\%) e parboilizado (13\%). Nunes et al. (2003), estudando amostras de arroz, observaram que o subgrupo mais contaminado foi o polido em relação ao parboilizado e ao integral. Segundo Amato et al. (2002), em relação à contaminação fúngica, o arroz parboilizado possui uma vantagem, pois, durante o encharcamento, em razão da ausência de ar por meio da imersão total em água evita-se que as espécies aflatoxigênicas proliferem. A única desvantagem é que no processo de gelatinização, como os grãos sofrem uma soldagem, pode ocorrer uma internalização da contaminação fúngica, pois, os microrganismos tendem a se acumular junto à fratura. Em arroz integral, em razão da presença enriquecida de nutrientes, a proliferação fúngica pode ser mais evidenciada, principalmente se os princípios básicos de secagem e armazenamento não forem devidamente realizados. Em alimentos orgânicos, tal como o arroz, de acordo com Bourn \& Prescott (2002), não há nenhuma evidência que possam ser mais suscetíveis à contaminação microbiológica que alimentos convencionais. Embora, neste trabalho, o maior índice de contaminação fúngica encontrado nas amostras de arroz foi detectado em orgânico. Pelo fato de não ser permitido o uso de fungicidas sintéticos no sistema orgânico, poderia haver uma maior possibilidade de produção de micotoxinas. Todavia, de acordo com Kouba (2002), estudos realizados até o momento não permitem afirmar que na agricultura orgânica a produção de micotoxinas seja maior. Nos dois sistemas (orgânico e convencional) o uso de boas práticas culturais e de estocagem dos alimentos permite reduzir o risco de contaminação com micotoxinas. As formas de evitar as contaminações fúngicas nos vários subgrupos de arroz residem, primordialmente, na adoção de boas práticas agrícolas de secagem e armazenamento conforme Pitt et al. (2000).

\section{Incidência de fungos toxigênicos}

A contaminação fúngica é importante não apenas sob o ponto de vista sensorial, mas também pelo perigo que a produção de micotoxinas representa para o consumidor Muninbazi \& Bullerman (1996). Na tabela 1, descrevem-se os resultados obtidos após a identificação das espécies. Foram identificados 184 isolados, predominando as espécies pertencentes aos gêneros Aspergillus e Penicillium. A. flavus se destaca por ter sido isolado dos quatro tipos de amostras.

Todas as espécies identificadas têm sido detectadas em diferentes tipos de arroz, conforme estudos realizados por Pitt \& Miscamble (1994), Lima et al. (2000) e Park et al. (2004). Hussaini et al. (2007) analisaram um total de 1062 isolados fúngicos identificados de 196 amostras de arroz da Nigéria. As principais espécies foram: Penicilium spp., A.flavus, A. parasiticus, A. niger e Rhizopus spp.

\section{Avaliação do Potencial toxigênico por Plug Ágar:}

A avaliação do potencial aflatoxigênico pelo teste Plug Agar demonstra que de 17 isolados de A. parasiticus testados, $14(82,35 \%)$ foram produtores de aflatoxinas $B_{1}$, $\mathrm{B}_{2}, \mathrm{G}_{1}$ e $\mathrm{G}_{2}$. Dos 31 isolados de $A$. flavus testados, 8 (25,80\%) foram produtores de aflatoxinas $\mathrm{B}_{1}$ e $\mathrm{B}_{2}$ Semelhante aos resultados obtidos por Prado et al (2009). A. flavus aflatoxigênicas estavam presentes em 6 amostras testadas, enquanto isolados de $A$. parasiticus aflatoxigênicos estavam presentes em 11 amostras testadas. Apesar de nem todas as linhagens de uma dada espécie serem capazes de produzir micotoxinas, a produção é bastante consistente em cultura pura, desde que se utilizem condições ótimas de produção.

\section{Características de Desempenho da Metodologia Analítica}

\section{Linearidade}

A linearidade foi avaliada na faixa de $0,4752 \mathrm{ng} \mathrm{mL}^{-1}$ a 79,2 $\mathrm{ng} \mathrm{mL}^{-1}$ para B1; 0,592 a 98,7 $\mathrm{ng} \mathrm{mL}^{-1}$ para $\mathrm{B} 2 ; 0,753$ a $125,57 \mathrm{ng} \mathrm{mL}^{-1}$ para G1 e 0,475 a $79,1 \mathrm{ng} \mathrm{mL}^{-1}$ para $\mathrm{G} 2$, e calculada a partir da equação da regressão linear, determinada pelo método dos mínimos quadrados. Foi utilizado o coeficiente de correlação linear $\left(\mathrm{R}^{2}\right)$ como indicador da reta como modelo matemático. Os valores sempre foram maiores que 0,99 como recomendado por Green (1996).

\section{Recuperação e Coeficiente de Variação}

Os resultados de recuperação obtidos (Tabela 2), em todos os níveis de fortificação efetuados, revelaram que a metodologia empregada está de acordo com o estabelecido pela Lei No401/2006 de 23/2/2006 da Commission Regulation (EC). 
Tabela 1 - Frequência das espécies contaminantes.

\begin{tabular}{|c|c|c|c|}
\hline Gênero/espécie & $\begin{array}{c}\text { Amostras } \\
\text { contaminadas }\end{array}$ & Tipos/ amostras & $\begin{array}{c}\text { N. isolados } \\
\text { identificados }\end{array}$ \\
\hline A. candidus & 8 & Polido (7) e Integral (1) & 22 \\
\hline A.carbonarius & 1 & Polido (1) & 1 \\
\hline A. flavus & 17 & $\begin{array}{c}\text { Polido(14); Orgânico (1) Integral(1) e } \\
\text { Parboilizado.(1) }\end{array}$ & 31 \\
\hline A. foetidus & 10 & Polido (9) e integral(1) & 12 \\
\hline A. niger & 7 & Polido (5), Integral (1) e Orgânico(1) & 14 \\
\hline A. oryzae & 8 & Polido (5) e Integral (3) & 10 \\
\hline A. parasiticus & 11 & Polido (9) Integral (2) & 17 \\
\hline A. versicolor & 3 & Integral (1),Orgânico.(1) e Polido.(1) & 6 \\
\hline A.fumigatus & 1 & Polido (1) & 1 \\
\hline A.niger Agregados & 10 & Polido (8) e Integral (2) & 14 \\
\hline A.ostianus & 3 & Polido (2) Integral (1) & 6 \\
\hline A.sulphureus & 1 & Polido (1) & 1 \\
\hline A.tamari & 2 & Polido (1) Integral (1) & 2 \\
\hline Alternaria alternata & 1 & Polido (1) & 1 \\
\hline $\begin{array}{l}\text { Aspergillus.spp. (Seção } \\
\text { Circumdati) }\end{array}$ & 1 & Polido (2) & 2 \\
\hline $\begin{array}{l}\text { Aspergillus.spp. (Seção } \\
\text { Nigri) }\end{array}$ & 1 & Orgânico (1) & 1 \\
\hline Cladosporium & 1 & Polido (1) & 1 \\
\hline Emericella nidulans & 4 & Polido (2) Integral (2) & 6 \\
\hline Eurotium & 1 & Polido (1) & 2 \\
\hline Fusarium spp. & 3 & Polido (1) e Integral (2) & 3 \\
\hline P.aurantiogriseum & 1 & Polido (1) & 1 \\
\hline P.brevicompactum & 1 & Polido (1) & 1 \\
\hline P.citrinum & 4 & Polido (4) & 4 \\
\hline P.funiculosum & 1 & Polido (1) & 1 \\
\hline P.glabrum & 2 & Polido (2) & 3 \\
\hline P.sclerotiorum & 1 & Polido (1) & 4 \\
\hline P.solitum & 2 & Polido (2) & 2 \\
\hline $\begin{array}{l}\text { Penicillium } \\
\text { griseofulvun }\end{array}$ & 3 & Polido (2) e Integral (1) & 3 \\
\hline Rhizopus spp. & 3 & Polido (1) Integral (2) & 7 \\
\hline Trichoderma spp. & 3 & Polido (3) & 3 \\
\hline
\end{tabular}

Tabela 2 - Valores de Recuperação recomendados pela Legislação européia.

\begin{tabular}{lccc}
\hline Aflatoxinas & Contaminações padrão & Recuperação padrão & Recuperação obtida (\%) \\
\hline AFB1, AFB2, AFG1, AFG2 & $<1 \mu \mathrm{g} / \mathrm{kg}$ & 50 a 120 & 65,4 a 99,8 \\
AFB1, AFB2, AFG1, AFG2 & $1-10 \mu \mathrm{g} / \mathrm{kg}$ & 70 a 110 & 70,9 a 90,8 \\
AFB1, AFB2, AFG1, AFG2 & $>10 \mu \mathrm{g} / \mathrm{kg}$ & 80 a 110 & 80 a 88,1 \\
\hline
\end{tabular}


Em relação aos coeficientes de variação, de acordo com Horwitz et al. (1980) devem ser menores que 30\% para indicarem eficiência do método. O limite de detecção baseado em 3 vezes o ruído da linha de base do aparelho é de $0,1 \mu \mathrm{g} \mathrm{kg}^{-1}$ e o limite de quantificação 3 vezes o limite de detecção $\left(0,3 \mu \mathrm{g} \mathrm{kg}^{-1}\right)$. O limite de detecção especificamente baseado em 3 vezes o ruído do sinal foi de 0,05 ng g-1 para G2, 0,04 para G1, 0,02 para B2 e 0,02 ng g-1 para B1. Os limites de quantificação foram considerados 10 vezes o ruído do sinal: G2: 0,16 ng g-1; G1: 0,11 ng g-1 ; B2: 0,05 ng g-1 e B1 0,07 ng g-1. Esses valores atendem à Resolução n.34/76 do Ministério da Saúde, que estabelece o limite máximo de $30 \mu \mathrm{g} \mathrm{kg-1} \mathrm{para}$ a soma de B1 e G1 (Brasil, 1977).

\section{Aflatoxinas em arroz}

De acordo com a Tabela 3, das 36 amostras de arroz somente uma estava contaminada com aflatoxina B1 (1,2 ìg $\left.\mathrm{Kg}^{-1}\right)$. Observa-se, também, que a presença de espécies potencialmente produtoras de aflatoxinas não indicou necessariamente a detecção de aflatoxinas nas amostras analisadas. Esses resultados sugerem que as espécies aflatoxigênicas não tiveram condições favoráveis para a produção de aflatoxinas.

Tabela 3 - Contaminação com Fungos Toxigênicos e Aflatoxina B1.

\begin{tabular}{|c|c|c|}
\hline Amostra & Resultado & $\begin{array}{c}\text { Fungos } \\
\text { aflatoxigênicos }\end{array}$ \\
\hline Amostra 1 a 3 & ND & - \\
\hline Amostra 4 & ND & A. parasiticus \\
\hline Amostra 5 & ND & A.parasiticus \\
\hline Amostra 6 & ND & A. parasiticus \\
\hline Amostra 7 e 8 & ND & - \\
\hline Amostra 9 & ND & A. parasiticus \\
\hline Amostra 10 & ND & - \\
\hline Amostra 11 & ND & A. parasiticus \\
\hline Amostra 12 & ND & $\begin{array}{c}\text { A. parasiticus } \\
\text { e A. flavus }\end{array}$ \\
\hline Amostra 13 a 15 & ND & - \\
\hline Amostra 16 & ND & A. parasiticus \\
\hline Amostra 17 a 20 & ND & - \\
\hline Amostra 21 a 34 & ND & A. flavus \\
\hline Amostra 35 & $\mathrm{~B} 1=1,2 \mu \mathrm{g} / \mathrm{kg}$ & A. parasiticus \\
\hline Amostra 36 & ND & A. flavus \\
\hline
\end{tabular}

ND: Não Detectado; Limite de Detecção; $0,1 \mu \mathrm{g} \mathrm{kg}^{-1}$; Limite de Quantificação: $0,3 \mu \mathrm{g} \mathrm{kg}^{-1}$.
Em estudos realizados na Índia por Reddy et al. (2008), das 1200 amostras analisadas, 67,8\% estavam contaminadas com níveis que variaram 0.1 a 308 ìg $\mathrm{Kg}^{-1}$. Segundo Bandara et al. (1991), analisando arroz no Siri Lanka, os níveis de AFB1 e AFG1 foram mais elevados em arroz parboilizado do que no arroz cru moído. A maior taxa de AFB1 foi $185 \mathrm{mg} \mathrm{Kg}^{-1} \mathrm{e}$ AFG1 de $963 \mathrm{mg} \mathrm{Kg}^{-1}$. A procedência dessas amostras é de uma região de umidade relativa $78 \%$ e temperatura média anual de $27^{\circ} \mathrm{C}$. Outros resultados têm demonstrado níveis mais baixos de contaminação. Segundo Park et al. (2004), a AFB1 estava presente em arroz coreano (5) das (88) amostras com níveis na faixa de 1,8 a 7,3 ìg/Kg. Conforme Simionato et al. (2003), analisando arroz comercializado no Brasil, de 68 amostras de arroz, duas de arroz polido tipo 1 continham AFB1 na faixa de 6 e 9 ìg $\mathrm{Kg}^{-1}$ e uma das amostras apresentou traços de aflatoxina B2. Silva et al (2008), analisando amostras provenientes do $5^{\circ}$ Batalhão de suprimento do Exercito Brasileiro em Curitiba (PR) observou que, de um total de 26 amostras analisadas, $6(23,07 \%)$ apresentaram positividade para aflatoxina B1 com níveis variando entre 0,54 a 2,04 ug $\mathrm{Kg}^{-1}$ e $1(3,84 \%)$ apresentou presença de aflatoxina B2. No Brasil, Prado et al. (1989) não detectaram a presença de aflatoxinas em arroz pelo procedimento descrito por Soares \& Rodrigues-Amaya (1988). A redução das micotoxinas pode ocorrer por processos térmicos. Para Park \& Kim (2006), no processo de cozimento comum do arroz usado na Coréia, com temperaturas em torno de $160^{\circ} \mathrm{C}$ verificou-se uma redução de 31-36\% da AFB1 em lotes naturalmente contaminados. Já, no processo de cozimento à pressão a $160^{\circ} \mathrm{C}$ por 20 minutos à pressão de $15 \mathrm{ib} /$ in houve redução de 78 a $88 \%$ da AFB1.

\section{CONCLUSÕES}

A metodologia empregada foi considerada adequada, tendo apresentado boa exatidão e precisão, demonstrando a viabilidade do método na quantificação de aflatoxinas em arroz. O nível de aflatoxina detectado em apenas uma amostra é considerado baixo. A presença de fungos toxigênicos em arroz reforça a necessidade de monitorar permanentemente a qualidade do produto em toda a cadeia produtiva.

\section{REFERÊNCIAS BIBLIOGRÁFICAS}

AMATO, G.W.; CARVALHO, J.L.V.; SILVEIRA, F.S.

Arroz parboilizado: tecnologia limpa, produto nobre. Porto Alegre: R.Lenz, 2002. 240p.

ARNOLDS, E.J.M. Biogeography and conservation. In: WICKLOW, D.T.; SODERSTROM, B. The mycota: envirnmental and microbial relationships. Berlin: Springer-Verlag, 1997. v.4, p.115-131. 
BANDARA, J.M.R.S.; VITHANEGE, A.K.I.; BEAN, G.A. Occurrence of aflatoxins in parboiled rice in Sri Lanka. Mycopathologia, Netherlands, v.116, p.65-70, 1991.

BOURN, D.; PRESCOTT, J. A comparison of the nutritional value, sensory qualities, and food safety of organically and conventionally produced foods. Critical Review Food Science Nutrition, Melbourne, v.42, n.1, p.1-34, jan. 2002.

BRASIL. Resolução 34/76, da Comissão de Normas e Padrões para Alimentos. Fixa padrões de tolerância para as aflatoxinas em alimentos. Diário Oficial da União, Brasília, 19 jan. 1977. Seção I, pt. I, p. 710.

FILTENBORG, O.; FRISVAD, J.C. A simple screening method for toxigenic moulds in pure cultures. Lebensmittel-Wissenschaft und -Technologie, London, v.13, p.128-130, 1980.

FRISVAD, J.C.; SAMSON, R.A. Filamentous fungi in foods and feeds: ecology, spoilage and mycotoxins production. In: ARORA, D.K.; MUKERJI, K.G.; MARTH, E.M. (Ed.). Handbook of applied mycology: foods and feeds. New York: M.Dekker, 1991. p.31-68.

FRISVAD, J.C.; SKOUBOB, P.; SAMSON, R.A. Taxonomic comparison of three different groups of aflatoxin producers and a new efficient producer of aflatoxin B1, sterigmatocystin and 3-O-methylsterigmatocystin, Aspergillus rambelli sp. Systematic and Applied Microbiology, London, v.28, p.442-453, 2005.

GREEN, J.M. A practical guide to analytical method validation. Analytical Chemistry, New York, v.68, n.9, p.305-309, 1996.

HORWITZ, W.; KAMPS, L.R.; BOYER, K.W. Quality assurance in the analysis of foods for trace constituents. Journal Association Official and Analytical Chemists, Washington, v.63, p.1344-54, 1980.

HUSSAINI, A.M.; TIMOTHY, A.G.; OLUFUNMILAYO, H.A.; SALAKO, E.A.; GODWIN, H.O. Fungi and some mycotoxins contaminating rice (Oriza sativa) in Niger State, Nigeria. African Journal of Biotecnology, Pretoria, v.6, n.2, p.99-108, 2007.

KLICH, M.A. Environmental and developmental factors influencing aflatoxin production by Aspergillus flavus and Aspergillus parasiticus. Mycoscience, Tokyo, v.48, p.1-80, 2007.

KLICH, M.A. Identification of Common Aspergillus species. The Netherlands: Centraalbureau vor Schimmelculture, 2002.

LIMA, C.A.; ORSI, R.B.; DILKIN, P.; CORRÊA, B. Mycoflora and aflatoxigenic in derivates of milled rice. Ciência $\mathrm{e}$ Tecnologia Alimentar, São Paulo, v.20, n.1, p.37- 39, 2000.

MUNINBAZI, C.; BULLERMAN, L. Molds and mycotoxins in foods from Burundi. Journal Food Protection, Washington, v.59, n.8, p.869-875, 1996.

NUNES, I.L.; MAGAGNI, G.; BERTOLIN, T.E.; FURLONG, E.B. Arroz comercializado na região sul do Brasil. Ciência e Tecnologia Alimentar, Campinas, v.23, n.2, p.190-194, maio/ago. 2003.

PARK, J.W.; KIM, Y.B. Effect of pressure on aflatoxin B1 in rice. Journal of Agricultural and Food Chemistry, Republic of Coreia, v.54, n.6, p.2431-2435, 2006.

PARK, J.W.; KIM, E.K.; KIM, Y.B. Estimation of the daily exposure of Koreans to aflatoxin B1 through food consumption. Food Additives and Contaminants, London, v.21, p.70-75, 2004.

PITT, J.I. A laboratory guide to common Penicillium species. 3.ed. Melbourne: Food Science, 2000.

PITT, J.I.; HOCKING, A.D. Fungi and food spoilage. 2.ed. Cambridgge: Chapman \& Hall, 1997. 593p.

PITT, J.I.; HOCKING, A.D.; BHUDHASAMAI, K.; MISCAMBLE, B.F.; WHEELER, K.A.; TANBOON, E.K. The normal mycoflora of commodities from Thailand: 2 , beans, rice, small grains and other commodities.

International Journal of Food Microbiology,

Netherlands, v.23, p.35-53, 1997.

PITT, J.I.; MISCAMBLE, B.F. Water relations of Aspergillus flavus and closely related species. Journal Food Protection, Washington, v.58, n.1, p.86-90, 1994.

PRADO, G.; MATTOS, S.V.M.; PEREIRA, E.C. Níveis de aflatoxinas em alguns alimentos consumidos em Belo Horizonte no período a 1988. Ciência e Tecnologia de Alimentos, v.9, n.2, p.138-147, jul./dez. 1989. 
PRADO, G.; ANDRADE, M. C. de; OLIVEIRA, M. S. de; LEAL, A. S.; OLIVEIRA, B. R. de; BATISTA, L. R. Efeito da irradiação na microbiota fúngica de plantas medicinais. Ciência e Agrotecnologia, Lavras, v. 33, n. 5, p. 1372-1378, set./out., 2009.

SAMSON, R.A.; HOEKSTRA, E.S.; FRISVAD, J.C.; FILTENBORG, O. Introdution to food-borne fungi. 4.ed. Berlin: Centralalbureau voor Schimmelcultures Baarn Deft, 2000.

SILVA, J.O. da; CANDIDO, L.M.B.; NOVELLO, D. Ocorrência de aflatoxinas em arroz consumido por militares do Exército Brasileiro por cromatografia em camada delgada e cromatografia líquida de alta eficiência. Ciência e Agrotecnologia, Lavras, v.32, n.4, p.1238-1244, 2008.

SIMIONATO, E.M.R.; ASTRAY, R.M.; SILOS, C.M. Ocorrência de ocratoxina A e aflatoxinas em arroz.
Revista do Instituto Adolfo Lutz, São Paulo, v.62, n.2, p.123-130, 2003.

SOARES, L.M.V.; RODRIGUES-AMAYA, D.B. Survey of aflatoxins, Ochratoxin A, Zearalenone, and Sterigmatocystin in some brazilian foods by using multitoxin thin-layer chromatographic method. Journal Association Official and Analytical Chemists, Washington, v.72, n.1, p.22-25, 1988.

STROKA, J.; ANKLAM, E.; JÖRISSEN, U.; GILBERT, J. Immunoaffinity column cleanup with liquid chromatography using post-column bromination for determination of aflatoxins in peanut butter, pistachio paste, fig paste, and paprika powder: collaborative study. Journal Association Official and Analytical Chemists International, n.83, p.320340, 2000. 